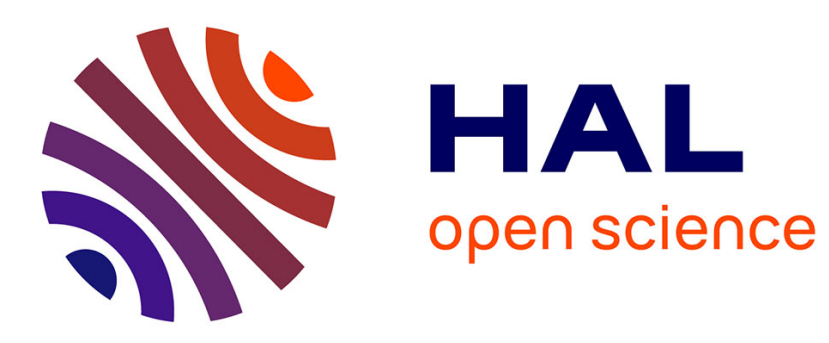

\title{
Gramian-based optimal design of a dynamic stroke amplifier compliant micro-mechanism.
}

\author{
Mathieu Grossard, Christine Rotinat-Libersa, Nicolas Chaillet
}

\section{To cite this version:}

Mathieu Grossard, Christine Rotinat-Libersa, Nicolas Chaillet. Gramian-based optimal design of a dynamic stroke amplifier compliant micro-mechanism.. IEEE/RSJ International Conference on Intelligent Robots and Systems, IROS'07., Oct 2007, San Diego, CA., United States. pp.4007-4012. hal-00187272

\section{HAL Id: hal-00187272 \\ https://hal.science/hal-00187272}

Submitted on 14 Nov 2007

HAL is a multi-disciplinary open access archive for the deposit and dissemination of scientific research documents, whether they are published or not. The documents may come from teaching and research institutions in France or abroad, or from public or private research centers.
L'archive ouverte pluridisciplinaire HAL, est destinée au dépôt et à la diffusion de documents scientifiques de niveau recherche, publiés ou non, émanant des établissements d'enseignement et de recherche français ou étrangers, des laboratoires publics ou privés. 


\title{
Gramian-based optimal design of a dynamic stroke amplifier compliant micro-mechanism
}

\author{
Mathieu Grossard, Christine Rotinat-Libersa, and Nicolas Chaillet
}

\begin{abstract}
This paper presents a new method developed for the optimal design of microrobotic compliant mechanisms. It is based on a flexible building block method, called FlexIn, which uses an evolutionary approach, to optimize a truss-like structure made of building blocks. From the first design step, in addition to conventional mechanical criteria, dynamic gramianbased metrics can be considered in the optimization procedure to fit expected frequency responses of the synthesized mechanisms. A planar monolithic compliant coupling structure is obtained by the optimal design method to act as a stroke amplifier for piezoelectric stacked actuators, to operate in both static and dynamic motions, and to passively filter out undesirable vibrations. Finally, performance comparisons between some of the pseudo-optimal FlexIn synthetized compliant mechanisms demonstrate the interests of the proposed optimization method for the design of dynamic operating smart microrobotic structures.
\end{abstract}

\section{INTRODUCTION}

$\mathrm{W}$ hen compared to other conventional actuation principles at small scales, piezoelectric ceramic actuators have very appealing properties in the sense of micromechatronic design. In particular, due to their high energy density, compactness, and high bandwidth, piezoceramic actuators are used for many high-frequency applications. Some meso-scale robots exploit the high bandwidth of piezoelectric actuators to achieve locomotion through an inchworm-like motion [1] or a stick-slip technique [2]. In [3], [4], they are applied to the design of microaerial vehicles, for which high energy density and efficiency of piezoelectric actuators are used to induce a flapping motion. Often, resulting piezoactuated devices are electromechanically tuned resonating microrobots. Moreover, piezoelectric actuation is mostly used for micro and nanorobot design in order to achieve nanometric and subnanometric resolutions in quasi-static mode [5]. However, for most piezoceramic materials, the induced strain is limited to about $0.1 \%$, so that a way of amplifying the actuator stroke is needed for most practical applications.

Manuscript received March 26, 2007.

M. Grossard is with both the Laboratoire d'Automatique de Besançon (LAB CNRS / ENSMM / UFC), Besançon, F-25000 France, and CEA LIST, Interactive Robotics Unit, Fontenay-aux-Roses, F-92265 France (email: mathieu.grossard@ens2m.fr).

C. Rotinat-Libersa is with the CEA LIST, Interactive Robotics Unit, Fontenay-aux-Roses, F-92265 France (e-mail: christine.rotinatlibersa@cea.fr).

$\mathrm{N}$. Chaillet is with the Laboratoire d'Automatique de Besançon (LAB CNRS / ENSMM / UFC), Besançon, F-25000 France (e-mail: nicolas.chaillet@ens2m.fr).
A number of papers address the problem of designing optimal coupling structures to act as stroke amplifiers for the piezoelectric actuator. Most often, these amplifying devices are compliant mechanisms, i.e. single-bodies, elastic continua flexible structures that transmit a motion by undergoing elastic deformation [6], as opposed to jointed rigid body motion of conventional articulated mechanisms. Using compliant mechanisms for the design of small scale systems is promising because of reduced kinematic noise, no wear, no backlash, simplified manufacturing, and ability to accommodate unconventional actuation schemes. A review of modelling and performance measures for stroke-amplified piezoceramic actuators and their application to compliant mechanism design is given in [7].

Previous works in topology design of coupling structures for piezoelectric actuators have mainly only focused on quasi-static applications, which may be sub-optimal in dynamic operations, or, worse, may induce degraded functioning. Very few related works deal with topological optimization method including dynamic analysis [8], [9], [10]. The objective functions generally use the maximization either of geometrical advantage (stroke amplification), or of mechanical efficiency, in the restrictive case of predetermined harmonic loadings. However, to adjust or enlarge the dynamic behavior of microrobotic structures, it can be very useful to optimize such devices taking into account versatile dynamic-based criteria from the first design step.

Therefore, we developed a more global systematic design approach, based on topology optimization of the structure, as well as that of their frequency responses, to design compliant micro-mechanisms [11]. This method is based on the flexible building block method, called FlexIn ("Flexible Innovation") [12]. To complete the panel of purely mechanical criteria, innovative gramian-based metrics are newly proposed in FlexIn. These criteria are useful tools to optimize dynamic operating flexible structures, and to ensure their efficient control afterwards. The possible interpretations of modal gramians for flexible structures in terms of mode controllability and observability [13] can be taken into account by different criteria in the optimization procedure. More generally, they can address several problems, such as avoiding noise amplification and decoupling disturbances [14], [15], avoiding spillovereffects [16], improving dynamic input/output performances [17], [18], which can be of great interest in the control oriented design of mechatronic devices.

This paper is organized as follows: firstly, we will briefly 
review the underlying idea of the FlexIn methodology for the design of smart compliant mechanisms. In a second part, we remind the typical form of balanced controllability and observability gramians, when the mechanism is discretized on its modal components. The last part aims at demonstrating the interests of some new gramian-based metrics proposed in FlexIn, in addition to static criteria, for the dynamic optimal synthesis of a stroke amplifier compliant device for a stacked piezoactuator.

\section{FLEXIN: A COMPLIANT MECHANISMS STOCHASTIC DESIGN METHODOLOGY}

In this section, we briefly present the flexible building block method FlexIn, which has been implemented for the optimal design of micromechanical planar mechanisms. The corresponding software developed with $M A T L A B^{\circledR}$ is also called FlexIn. It uses a multi-objective evolutionary algorithm approach for the optimal design of smart compliant mechanisms, which can be made either of an assembly of elementary passive or active compliant building blocks, chosen in two specific libraries. More detailed descriptions of the method can be found in [11], [19], [20].

\section{A. Compliant building blocks}

Two libraries of compliant elements in limited number are proposed in FlexIn. These bases are composed respectively of 36 and 19 elements of passive and piezoactive blocks, made of beams assembly (Fig. 1). They are sufficient to build a high variety of topologies. In particular, the various topologies of piezoactive blocks allow them to furnish multiple coupled degrees of freedom, thus generating more complex movements with only one building block. Moreover, the block feasibility related to fabrication process constraints can also be taken into account at this stage.

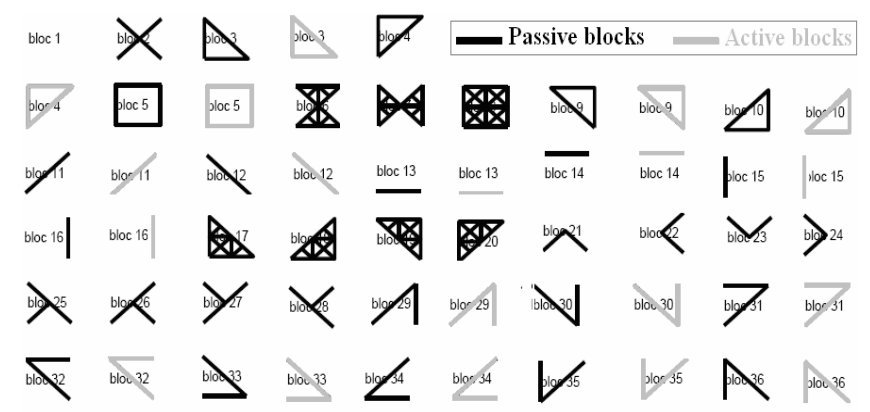

Fig. 1. Passive (black) and active (grey) libraries of compliant building blocks, for planar compliant mechanisms synthesis using FlexIn.

\section{B. Principles of the method and design parameters}

The purpose of FlexIn is to optimally design realistic compliant structures. The specification of a planar compliant mechanism problem considers specific boundary conditions: fixed frame location, input (actuators), contacts and output (end-effector). Different types of actuation principles can be used: either external or internal force/displacement actuators defined at particular nodes of the mesh [11], or integrated piezoactive elements taken from the active library [19]. The design method consists of searching for an optimal distribution of allowed building blocks, as well as for the optimal set of structural parameters and materials. The location of fixed nodes and that of the actuators and/or piezoactive blocks can also be considered as optimization parameters. The topology optimization method, inspired from [21], uses a genetic algorithm approach, which allows a true multicriteria optimization and the use of discrete variables (Fig. 2). The algorithm is structured as follows:

- Discrete variable parameterization of compliant mechanisms considering conception requirements (mesh size, topology, material and thickness, boundary conditions),

- Evaluation of individuals (design criteria calculation),

- Stochastic operators for the optimization (modification of compliant mechanisms description).

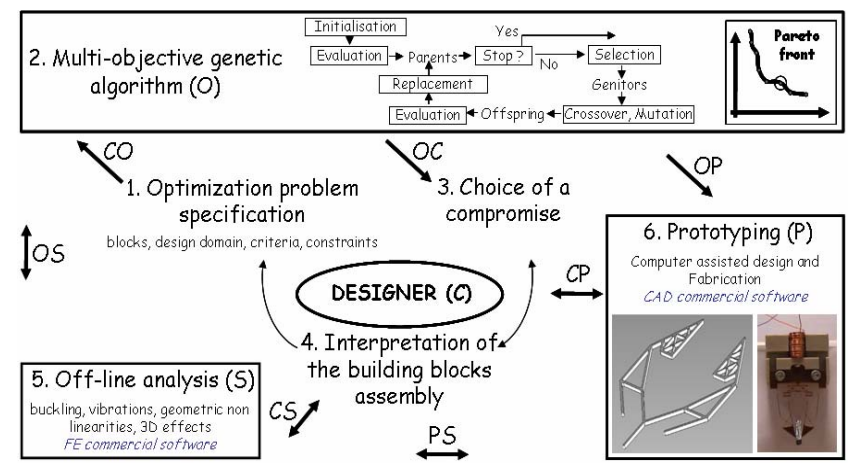

Fig. 2. Flowchart of the FlexIn optimal design method of compliant structures (multicriteria optimization).

\section{Multi criteria genetic algorithm}

Many fitness functions are available in FlexIn, thus allowing the optimal design of devices within a wide schedule of conditions. Several mechanical fitness can be specified for the optimization problem: free displacement and blocking force at the output port, strain energy (SE), mutual strain energy (MSE), maximal stress (yield or fatigue strength), geometric advantage (GA), mechanical advantage (MA), i.e. force amplification, etc. To meet specific control requirements for microrobotics devices design using FlexIn, various metrics based on modal gramians interpretations, have also been specified as possible fitness. Obviously, the design strategy depends on the metrics chosen, which must be based on the real needs of the device use. Let us note that multi-degrees of freedom mechanisms design can also be considered.

The optimization algorithm generates a set of pseudooptimal solutions (see 2 in Fig. 2) in the case of multicriteria optimization (and obviously only one global optimal solution for monocriterion optimization). The designer can next choose, analyse and interpret the obtained structures that best suit his design problem (see 3 to 5 in Fig. 2). 


\section{USEFul MEASURes of JoInt CONTROLlability AND}

OBSERVABILITY FOR THE OPTIMIZATION PROCEDURE

From the computation of the linear dynamic state model of the studied system, an optimal topology design strategy is derived taking into account control criteria, to meet inputoutput transfer performances with specific operations requirements. The joint controllability and observability study of resonant modes is a useful way to characterize the system in dynamic operations. Two numerical criteria based on eigenvalues of balanced gramian will be defined to help designing a dynamically efficient amplifier mechanism.

\section{A. State model of structures under FlexIn formalism}

In FlexIn, it is assumed that the compliant mechanisms are undergoing structural deformations, mainly due to the bending of the beams constituting the blocks. The models of the blocks are firstly obtained considering Navier-Bernoulli beam type finite elements. Structural parameters of each rectangular block are height, width, thickness, and material characteristics. The stiffness and mass matrices of each block are then calculated numerically, considering every combination of the discrete values allowed for the structural optimization variables. The calculation of the different matrices of each valued-block is done one time only at the beginning of the optimal design problem (before running the genetic algorithm), thus saving calculus time. The global dynamic behaviour of a structure results from the mass and stiffness matrices assembly of the constitutive blocks, and is done at each step for each individual during the optimization process. As in [22], each FlexIn structure is defined as a finite-dimensional, controllable and observable linear system with small damping and complex conjugate poles. Its nondamped form is represented by the following second-order matrix differential equations:

$$
\begin{aligned}
& \mathbf{M} \ddot{\boldsymbol{\eta}}+\mathbf{K} \boldsymbol{\eta}=\mathbf{E u} \\
& \mathbf{y}=\mathbf{F} \boldsymbol{\eta}
\end{aligned}
$$

Let us consider the integers $\mathrm{p}, \mathrm{s}$, and $\mathrm{r}$, which denote the numbers of degrees of freedoms (DOF) of the structure, of inputs and of outputs respectively. In (1) and (2), $\boldsymbol{\eta}$ is the $\mathrm{p} \times 1$ nodal displacement vector, $\mathbf{u}$ is the $\mathrm{s} \times 1$ input vector, $\mathbf{y}$ is the $r \times 1$ output vector. The $p \times p$ mass and stiffness matrices are denoted $\mathbf{M}>0$ and $\mathbf{K} \geq 0$, the input matrix $\mathbf{E}$ is $\mathbf{p} \times \mathbf{s}$ and the output displacement matrix $\mathbf{F}$ is $r \times p$. Each element of $\mathbf{u}$ (resp. y) denotes a physical actuator (resp. sensor) whose DOF location is defined by the location of the nonzero entry in the corresponding column in $\mathbf{E}$ (resp. rows in $\mathbf{F}$ ).

The harmonic solutions of (1) give the eigenvectors matrix $\boldsymbol{\Psi}$ and eigenfrequencies $\omega_{i}$. Replacing $\boldsymbol{\eta}$ by $\boldsymbol{\Psi q}$, where $\mathbf{q}$ is the $\mathrm{p} \times 1$ modal displacement vector, and multiplying Eq. (1) on the left by $\boldsymbol{\Psi}^{\mathrm{T}}$, the induced orthogonality relationships in modal form, lead to:

$$
\ddot{\mathbf{q}}+\operatorname{diag}\left(\omega_{\mathrm{i}}^{2}\right) \mathbf{q}=\boldsymbol{\Psi}^{\mathrm{T}} \mathbf{E} \mathbf{u}, \quad \mathbf{y}=\mathbf{F} \Psi \mathbf{q} .
$$

One possible state vector $\mathbf{x}$, of dimension $2 p \times 1$, consists of modal velocities and frequency weighted modal displacements:

$$
\mathbf{x}=\left(\begin{array}{lllll}
\dot{\mathrm{q}}_{1} & \omega_{1} \mathrm{q}_{1} & \ldots & \dot{\mathrm{q}}_{\mathrm{p}} & \omega_{\mathrm{p}} \mathrm{q}_{\mathrm{p}}
\end{array}\right)^{\mathrm{T}} .
$$

The analytical advantages of this choice are mentioned in [23]. Thus, (3) becomes (5), where the triplet (A, B, C) denotes the modal state-space representation of a structure:

$$
\dot{\mathbf{x}}=\mathbf{A x}+\mathbf{B u}, \quad \mathbf{y}=\mathbf{C} \mathbf{x}
$$

The matrices take the forms $\mathbf{A}=\operatorname{diag}\left(\mathbf{A}_{1}, \ldots, \mathbf{A}_{\mathbf{p}}\right)$, $\mathbf{B}=\left(\begin{array}{lll}\mathbf{B}_{\mathbf{1}}^{\mathbf{T}}, & \ldots, & \mathbf{B}_{\mathbf{p}}^{\mathbf{T}}\end{array}\right)^{\mathrm{T}}$, and $\mathbf{C}=\left(\mathbf{C}_{\mathbf{1}}, \ldots, \quad \mathbf{C}_{\mathbf{p}}\right)$, with:

$$
\mathbf{A}_{\mathbf{i}}=\left[\begin{array}{cc}
-2 \zeta_{\mathrm{i}} \omega_{\mathrm{i}} & -\omega_{\mathrm{i}} \\
\omega_{\mathrm{i}} & 0
\end{array}\right], \quad \mathbf{B}_{\mathbf{i}}=\left[\begin{array}{c}
\mathrm{b}_{\mathrm{i}} \\
0
\end{array}\right], \quad \mathbf{C}_{\mathbf{i}}=\left[\begin{array}{ll}
0 & \frac{\mathrm{c}_{\mathrm{i}}}{\omega_{\mathrm{i}}}
\end{array}\right],
$$

where $b_{i}$ and $c_{i}$ are the $i^{\text {th }}$ components of $\boldsymbol{\Psi}^{\mathbf{T}} \mathbf{E}$ and $\mathbf{F} \boldsymbol{\Psi}$ respectively, and $\zeta_{\mathrm{i}}$ is the modal damping ratio determined experimentally. It is important to note that $\mathbf{A}$ matrix depends on the structure itself (eigenfrequencies and damping ratio), $\mathbf{B}$ matrix on the location and actuators class, and $\mathbf{C}$ matrix on location and sensors class.

\section{B. Computation of the balanced Gramian}

Controllability $\left(\mathbf{W}_{\mathbf{c}}\right)$ and observability $\left(\mathbf{W}_{\mathbf{0}}\right)$ gramians are convenient forms to characterize system controllability and observability. They are obtained from solution of the following Lyapunov equations:

$$
\begin{aligned}
& \mathbf{A} \mathbf{W}_{\mathbf{c}}+\mathbf{W}_{\mathbf{c}} \mathbf{A}^{\mathrm{T}}+\mathbf{B B} \mathbf{B}^{\mathrm{T}}=\mathbf{0} \\
& \mathbf{A}^{\mathrm{T}} \mathbf{W}_{\mathbf{o}}+\mathbf{W}_{\mathbf{0}} \mathbf{A}+\mathbf{C}^{\mathrm{T}} \mathbf{C}=\mathbf{0}
\end{aligned} .
$$

For stable $\mathbf{A}$ and minimal $(\mathbf{A}, \mathbf{B}, \mathbf{C})$, solutions are positive definite, and the geometric interpretations are well understood [13]. The balanced case, where controllability and observability gramians are diagonal and equal to Hankel singular values (HSV) gramian $\Gamma^{2}=\operatorname{diag}\left(\gamma_{i}^{2}\right)$, is a useful tool for quantifying the joint controllability and observability of a system, because it characterizes efficient outputs control by the inputs. Assuming small damping and separated poles, the modal and balanced representations of flexible structures are closely related [13]. The physical modal state coordinates are approximately balanced in this asymptotic situation, and the approximate $\mathrm{i}^{\text {th }}$ Hankel singular value $(\mathrm{i}=1$, $\ldots$, p) for flexible structure is given by:

$$
\gamma_{\mathrm{i}}^{2}=\frac{\mathrm{b}_{\mathrm{i}} \mathrm{c}_{\mathrm{i}}}{4 \zeta_{\mathrm{i}} \omega_{\mathrm{i}}^{2}},
$$

where $\mathrm{i}$ denotes the indice of each structural mode. In the context of flexible system design, it is important to note that joint controllability and observability of a particular mode 
depends on its frequency, its damping effect, and its actuator and sensor joint contribution to the structure through the modal coefficient $\mathrm{b}_{\mathrm{i}} \mathrm{c}_{\mathrm{i}}$.

\section{Two gramian-based criteria proposed for compliant mechanism dynamic optimization}

We consider in this paper a study whose aim is to design a stroke amplifier compliant mechanism (see IV) which can address two problems often encountered in these applications.

1) Resonant modes rejection: To dynamically control outputs with the inputs in open-loop operations, resonance phenomenon have to be avoided because they can cause changes in stroke amplification and in phase angle response with the actuation frequency. Given a fixed input/output location, the optimal topologies are firstly chosen as the ones which guarantee the lowest joint controllability and observability for the first resonant modes. Thus, remoteness of resonance occurrences from low-frequency spectrum can be performed through the maximization of the following criteria $\mathrm{J}_{1}$, expressed in logarithmic scale for numerical convenience:

$$
\mathrm{J}_{1}=\sum_{1 \leqslant \exists_{1}} \frac{1}{\log _{10}\left(10+\gamma_{\mathrm{i}}^{2}\right)},
$$

where $I_{1}=\min \left(i / \gamma_{i}^{2} \neq 0\right)$. This numerical criterion gives preference to structures topology for which the first structural resonant modes are not detected, i.e. $\gamma_{i}^{2}=0$ for $1 \leq \mathrm{i}<\mathrm{I}_{1}$, in the transfer from input $\mathrm{u}$ to controlled output $\mathrm{y}$. Thus, first resonance occurrence, denoted by HSV $\gamma_{\mathrm{I}_{1}}^{2}$, is of higher frequency, while being at the same time attenuated in magnitude.

2) Filtering out undesirable vibrations: Vibration reduction can be considered as attenuating the modal transfer of disturbing dynamic inputs $\mathrm{w}$ transmitted from the fixed base nodes through B' matrix to output y. The corresponding plant has the following state-space realisation:

$$
\dot{\mathbf{x}}=\mathbf{A x}+\mathbf{B u}+\mathbf{B}^{\prime} \mathbf{w}, \quad \mathbf{y}=\mathbf{C x}
$$

From the above (A, B', C) triple, balanced gramian $\Gamma^{\prime 2}=\operatorname{diag}\left(\gamma_{\mathrm{i}}^{\prime 2}\right)$ that governs dynamics controllability from the disturbances to controlled outputs, is computed. $\mathrm{J}_{2}$ criteria, to be maximised, is introduced to avoid noise amplification, with the constraint of guaranteeing some minimum modal controllability and observability on each frequency mode in a certain bandwidth of interest $\left[\omega_{\mathrm{m}}, \omega_{\mathrm{M}}\right]$,

$$
\mathrm{J}_{2}=\sum_{\mathrm{i} \in \mathrm{I}_{2}} \frac{1}{\log _{10}\left(10+\gamma_{\mathrm{i}}^{\prime 2}\right)},
$$

where $I_{2}=\left\{i / \omega_{m} \leqslant \omega_{i} \leqslant \omega_{M}\right\}$. Topology structures with only few resonant vibration modes in the desired bandwidth, moved away from $\omega_{\mathrm{m}}$ and attenuated preferably, will be privileged by $\mathrm{J}_{2}$ criteria.

\section{OPTIMAL DESIGN OF A STROKE AMPLIFICATION COMPLIANT MICROMECHANISM FOR STACKED PIEZOACTUATOR}

\section{A. Optimization problem specification}

The problem assumed is: a $2 \mu \mathrm{m}$-stroke along $\mathrm{x}$ axis (see Fig. 3) piezoceramic PICMA Multilayer actuator (from PI Ceramic Technology [24]) has to be used together with a mechanical amplifier. The compliant coupling structure is supposed to be made of SU8-resin material (Table I). The half part of the symmetrical micromechanism is considered to have a maximal size of $4 \mathrm{~mm} \times 2 \mathrm{~mm}$ (Fig. 3). For the optimal synthesis run, the number of building blocks in the half compliant mechanism is fixed to six. When an actuation displacement is applied to the input port by the stacked actuator, the output end-effector node of the amplifier has to move along the $\mathrm{x}$ axis. The thickness of the planar structure is taken constant and equal to $500 \mu \mathrm{m}$. The topology and size of the blocks are the parameters to optimize. The used blocks are only the passive ones defined in Fig. 1. This topological optimization problem allows more than $10^{17}$ candidate structures.

Geometric advantage (GA), $J_{1}$ and $J_{2}$ gramian-based criteria (with $\omega_{\mathrm{m}}=0 \mathrm{rad} / \mathrm{s}, \omega_{\mathrm{M}}=250,000 \mathrm{rad} / \mathrm{s}$ ), are the objective functions to maximize simultaneously.

TABLE I.

MECHANICAL CHARACTERISTICS OF SU8-RESIN

\begin{tabular}{cc}
\hline \hline Young modulus & $\mathrm{E}=4020 \mathrm{Mpa}$ \\
\hline Poisson ratio & $\nu=0.22$ \\
\hline Density & $\rho=1.19$ \\
\hline Modal damping ratio & $\zeta=0.5 \%$ \\
\hline
\end{tabular}

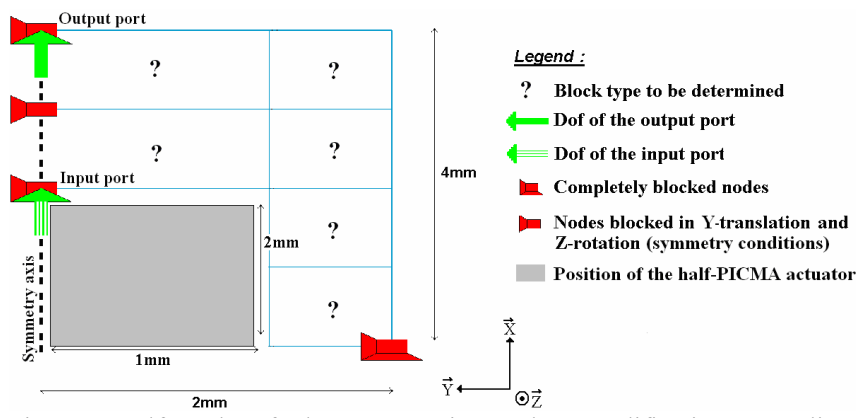

Fig. 3. Half-mesh of the symmetric stroke amplification compliant mechanism with imposed boundary conditions.

\section{B. Results}

The optimal synthesis method can generate stroke amplifier mechanisms for the piezoelectric actuator. The best compromise structures are kept, when the genetic algorithm does not find any new pseudo-optimum during 200 subsequent generations. The set of pseudo-optimal solutions can be represented on Pareto fronts (Fig. 4). From these fronts, three selected pseudo-optimal solutions, referred as $\mathrm{A}, \mathrm{B}$ and $\mathrm{C}$ structures in the following, will illustrate performance comparison (Table II). Structure A exhibits good quasi-static performances (high GA value), whereas structures 
$\mathrm{B}$ and $\mathrm{C}$ have good dynamic fitness (high $\mathrm{J}_{1}$ and $\mathrm{J}_{2}$ values), particularly structure $\mathrm{C}$ for $\mathrm{J}_{2}$ criteria.
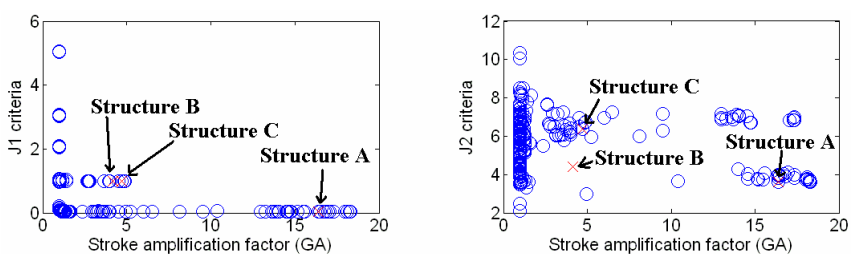

Fig. 4. Pareto fronts of compliant mechanisms synthesized using FlexIn (genetic parameters used: population of 100 individuals, mutation probability of $40 \%$ on genes and individuals).

TABLE II. FLEXIN REPRESENTATION AND PERFORMANCES OF THE A, B AND C COMPLIANT STROKE AMPLIFIERS

\begin{tabular}{|c|c|c|c|c|}
\hline \multirow{2}{*}{$\begin{array}{l}\text { Pseudo- } \\
\text { optimal } \\
\text { amplifier }\end{array}$} & \multirow{2}{*}{$\begin{array}{l}\text { Topology of compliant } \\
\text { structures }\end{array}$} & \multicolumn{3}{|c|}{ Criteria values } \\
\hline & & GA & $\mathrm{J}_{1}$ & $\mathrm{~J}_{2}$ \\
\hline A & & 16.38 & 0.04 & 3.59 \\
\hline B & & 4.15 & 1.00 & 4.40 \\
\hline & 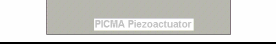 & & & \\
\hline $\mathrm{C}$ & & 4.70 & 0.99 & 6.38 \\
\hline & & & & \\
\hline
\end{tabular}

\section{Dynamic performances comparison}

1) Resonant modes treatment: Frequency characteristic analysis between input $\mathrm{u}$ and output $\mathrm{y}\left(\mathrm{J}_{1}\right.$ criteria $)$ is important to understand the dynamic performances of the compliant stroke amplifiers (Fig. 5), especially when they have a wide range of working frequencies. As expected by poor $J_{1}$ criteria value for designed "favourite" quasi-static stroke amplifier $\mathrm{A}$, the occurrence of the first frequency resonance phenomenon $\left(\omega_{\mathrm{A}}=45,976 \mathrm{rad} / \mathrm{s}\right)$ is low, and excludes its use for open-loop operations at high frequencies. Indeed, in those dynamic operations, the geometric advantage exhibits important changes in magnitude, and the phase difference is shifted near $180^{\circ}$, i.e. the input and the output are moving in opposite directions, which is not the intended performance. On the contrary, rejected first resonance of the $\mathrm{B}$ and $\mathrm{C}$ amplification mechanisms $\left(\omega_{B}=338,260 \mathrm{rad} / \mathrm{s}\right.$ and $\left.\omega_{C}=149,380 \mathrm{rad} / \mathrm{s}\right)$ allow a dynamic use over a larger range of low-frequencies (as expected by high $\mathrm{J}_{1}$ criteria values).
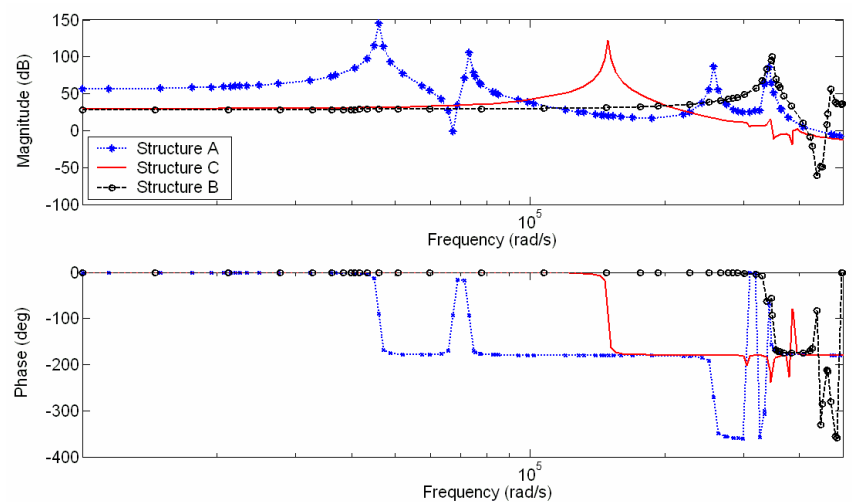

Fig. 5. GA frequency responses of $\mathrm{A}, \mathrm{B}$, and $\mathrm{C}$ structures between input $\mathrm{u}$ and output $y$ in low-frequency spectrum.

2) Undesirable vibrations treatment: Vibration reduction between the disturbances from fixed frame inputs $\mathrm{w}$ and the outputs y in open-loop operations ( $\mathrm{J}_{2}$ criteria) is also made in a global sense by limiting in number, attenuating, or, better, remoting from the bandwidth of interest, resonant modes (Fig. 6).
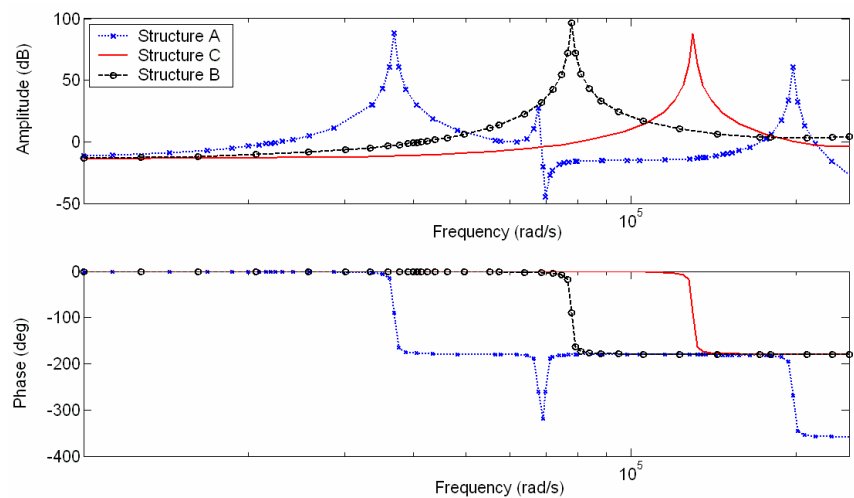

Fig. 6. Frequency responses of A, B, and C structures between disturbance input $\mathrm{w}$ and output $\mathrm{y}$ in the bandwidth of interest.

Considering $\mathrm{J}_{2}$ criteria, structure $\mathrm{C}$ is the most appropriate one (high $\mathrm{J}_{2}$ ) to passively deal with undesirable vibrations in the spectrum $\left[\omega_{\mathrm{m}}=0 \mathrm{rad} / \mathrm{s}, \omega_{\mathrm{M}}=250,000 \mathrm{rad} / \mathrm{s}\right]$. In that lowfrequency range, it contains only one resonance phenomenon, which has the highest frequency value $\omega_{C}=129,920 \mathrm{rad} / \mathrm{s}$ compared to first resonant modes of $\mathrm{A}$ and $\mathrm{B}$ structures. Indeed, structure A (low $\mathrm{J}_{2}$ ) has three resonant modes in that spectrum of interest, $\omega_{\mathrm{Al}}=36,968 \mathrm{rad} / \mathrm{s}$, $\omega_{\mathrm{A} 2}=67,847 \mathrm{rad} / \mathrm{s}, \omega_{\mathrm{A} 3}=197,960 \mathrm{rad} / \mathrm{s}$, and structure $\mathrm{B}$ exhibits one unique resonance mode $\omega_{\mathrm{B}}=77,818 \mathrm{rad} / \mathrm{s}$, but characterized with a lower value than $\omega_{\mathrm{C}}$.

\section{CONCLUSION}

A new concept of optimal design method for compliant static 
and dynamic operating micro-mechanisms has been presented, considering gramian-based criteria, in addition to classical mechanical ones. In this method, called FlexIn, smart compliant mechanisms are made of an assembly of building blocks. Complex multi-objective design problems can be solved by FlexIn to take advantage of versatile criteria to synthesize high performances microrobotic compliant mechanisms designs.

Open-loop dynamic based considerations lead to two efficient numerical criteria $\mathrm{J}_{1}$ and $\mathrm{J}_{2}$, depending on chosen eigenvalues of the balanced gramian matrix. It has been shown that the use of these gramian-based criteria helps designing mechanisms, by giving information about their frequency response over a spectrum of interests, to meet specific dynamic or control requirements. In the example studied in this paper, the resulting pseudo-optimal solutions can successfully operate at both static and low-frequency dynamic regimes, and simultaneously filter out unwanted vibrations.

Prototypes of the three compliant micromechanisms, resulting from the optimal design with FlexIn, will be realized soon for experimental validations. Gramian criteria will also be taken into account for designing other smart structures with embedded piezoactive building blocks, and to include the actuator dynamics as well.

\section{REFERENCES}

[1] Jr. May, G. William, "Piezoelectric electromechanical Translation apparatus", US Patent No. 3902084, 1975.

[2] S. Fablbusch, and al., "Flexible Microrobotic System MINIMAN: Design, Actuation Principle and control", Proc. of the IEEE/ASME International Conference on Advanced Intelligent Mechatronics, pp. 156-161, 1999.

[3] A. Cox, E. Garcia, and M. Goldfarb, "Actuator development for a flapping microrobotic microaerial vehicle," SPIE Microrobotics Symposium, Boston, MA, Nov. 1998.

[4] M. Setti, "Piezoelectrically actuated four-bar mechanism with two flexible links for micromechanical flying insect thorax", IEEE/ASME Transactions on Mechatronics, Vol. 8, No. 1, 2003.

[5] J. Agnus, P. Nectoux, N. Chaillet, "Overview of microgrippers and micromanipulation station based on a MMOC microgripper", Proc. of the IEEE International Symposium on Computational Intelligence in Robotics and Automation, pp. 117-123, 2005.

[6] M. Frecker, S. Canfield, "Optimal design and experimental validation of compliant mechanical amplifiers for piezoceramic stack actuators", Journal of Intelligent Material Systems and Structures, Vol. 11, pp. 360-369, 2000.

[7] M. Abdalla, and al., "Design of a piezoelectric actuator and compliant mechanism combination for maximum energy efficiency", Smart Material and Structures, Vol. 14, pp. 1421-1430, 2005.

[8] H. Maddisetty, M. Frecker, "Dynamic topology optimization of compliant mechanisms and piezoceramic actuators", ASME Journal of Mechanical Design, Vol. 126, Iss. 6, pp. 975-983, 2002.

[9] H. Du, and al., "Topological optimization of mechanical amplifiers for piezoelectric actuators under dynamic motion", Smart Material and Structures, Vol. 9, pp. 788-800, 2000.

[10] G. K. Lau, and al., "Systematic design of displacement-amplifying mechanisms for piezoelectric stacked actuators using topology optimization", Journal of Intelligent Material Systems and Structures, Vol. 11, pp. 685-695, 2000.

[11] M. Grossard, C. Rotinat-Libersa, N. Chaillet, "Redesign of the MMOC microgripper piezoactuator using a new topological method", IEEE/ASME International Conference on Advanced Intelligent Mechatronics, Zürich, Switzerland, 2007, accepted for publication.
[12] P. Bernardoni, and al., "A new compliant mechanism design methodology based on flexible building blocks", Smart Material and Structures, USA, Vol. 5383, pp. 244-254, 2004.

[13] E. A. Jonckheere, "Principal components analysis of flexible systems Open-loop case", IEEE Trans. on Automatic Control, Vol. 29, No. 12, pp. 1095-1097, 1984.

[14] M. R. Kermani, M. Moallem, R. V. Patel, "Optimizing the performance of piezoelectric actuators for active vibration control", in Proc. of IEEE International Conference on Robotics and Automation, Washington DC, pp. 2375-2380, 2002.

[15] W. Chen, and al., "Optimal sensor design and control of piezoelectric laminate beams", IEEE Trans. on Control Systems and Technology, Vol. 12, No. 1, pp. 148-155, 2004.

[16] M. Collet, "Shape optimization of piezoelectric sensors dealing with spill-over instability", IEEE Trans. on Control Systems Technology, Vol. 9, No. 4, pp. 654-662, 2001.

[17] M. R. Kermani, , R. V. Patel, M. Moallem, "Flexure control using piezostack actuators: design and implementation", IEEE/ASME Trans. on Mechatronics, Vol. 10, No. 2, pp. 181-188, 2005.

[18] W. Liu, Z. Hou, M. A. Demetriou, "A computational scheme for the optimal sensor/actuator placement of flexible structures using spatial H2 measures", Mechanical Systems and Signal Processing, Vol. 20, pp. 881-895, 2006.

[19] M. Grossard M., C. Rotinat-Libersa, N. Chaillet, Y. Perrot, "Flexible building blocks method for the optimal design of compliant mechanisms using piezoelectric material", $12^{\text {th }}$ IFToMM World Congress, Besançon, France, paper n ${ }^{\circ} 684,2007$.

[20] C. Rotinat-Libersa, Y. Perrot, J.-P. Friconneau, "Potentialities of optimal design methods and associated numerical tools for the development of new micro- and nano- intelligent systems based on structural compliance - An example -", IARP-IEEE/RAS-EURON JointWorkshop on Micro and Nano Robotics, Paris, France, 11p, 2006.

[21] K. Deb, and al., "A fast elitist non-dominated sorting genetic algothm for multi-objective optimization: Nsga-II", Proc. of the 6th Int. Conf. on Parallel Problem Solving from Nature, France, pp. 849-858, 2000.

[22] K. B. Lim, W. Gawronski, "Actuators and sensor placement for control of flexible structures", in Control and Dynamics Systems: Advances in Theory and Applications, ed. London, Academic Press, 1993.

[23] A. Hac, and L. Liu, "Sensor and actuator location in motion control of flexible structures", J. Sound Vib., Vol. 167, No. 2, pp. 239-261, 1993.

[24] PI Piezo Ceramic Technology, 2005. Available: http://www.piceramic.com/ 